\title{
Improving diagnosis and treatment of osteoporosis: evaluation of a clinical pathway for low trauma fractures
}

\author{
L. L. Laslett • J. N. Whitham • C. Gibb • \\ T. K. Gill • J. A. Pink • J. D. McNeil • \\ on behalf of the Osteoporosis SA Refracture Prevention \\ Pathway Steering Committee
}

Received: 5 February 2007 / Accepted: 16 February 2007 / Published online: 29 March 2007

(C) International Osteoporosis Foundation and National Osteoporosis Foundation 2007

\begin{abstract}
Summary Diagnosis and treatment of osteoporosis in hospitals is poor. We compared patient outcomes before and after implementation of a clinical protocol for lowtrauma fractures. Patients in the pathway were more likely to receive information about osteoporosis or osteoporosis medications. Therefore our clinical pathway is effective in improving osteoporosis information and treatment.

Introduction Effective therapies for reducing fracture risk are available, yet under-utilised in hospital settings. We aimed to increase rates of initiation of osteoporosis investigations, pharmacological treatment, treatment continuation, and follow-up general practitioner (GP) visits.

Methods Comparison of patient outcomes before and after implementation of a clinical pathway in patients admitted
\end{abstract}

L. L. Laslett $(\bowtie) \cdot$ J. D. McNeil

The University of Adelaide Discipline of Medicine,

Modbury Public Hospital,

Smart Road,

Modbury, South Australia 5092, Australia

e-mail: laura.laslett@adelaide.edu.au

J. N. Whitham

Research and Evaluation Unit,

Women's and Children's Hospital,

Adelaide, South Australia, Australia

C. Gibb

Department of Orthopaedics, The Queen Elizabeth Hospital,

Adelaide, South Australia, Australia

T. K. Gill

Population Research and Outcomes Studies Unit,

South Australian Department of Health,

Adelaide, South Australia, Australia

J. A. Pink

Department of Pharmacy, The Queen Elizabeth Hospital,

Adelaide, South Australia, Australia for low-trauma fractures to the Department of Orthopaedics and Trauma at The Queen Elizabeth Hospital, Adelaide. Results Patients enrolled in the osteoporosis clinical pathway $(\mathrm{n}=28)$ were more likely than patients receiving usual care $(\mathrm{n}=28)$ to have received information about $(54 \%$ vs. $29 \% ; \mathrm{p}<0.05$ ), or a prescription for osteoporosis medication $(53 \%$ vs. $25 \%, \mathrm{p}<0.05)$. Differences in proportions of patients visiting their GP post fracture and in osteoporosis investigations suggested or undertaken were not significant. At the later audit, the high proportion of patients receiving information about osteoporosis medication had been maintained $(51 \%)$. Prescription of osteoporosis medications increased to $83 \%(p<0.01)$, and more patients saw their GP post fracture $(87 \% ; p<0.01)$. High rates of medication adherence were reported in patients in all groups receiving prescriptions.

Conclusion A clinical pathway for improving hospital management of osteoporosis is effective in improving education about, prescription for, and uptake of osteoporosis medications.

Keywords Osteoporosis · Clinical protocols .

Bone density . Fractures

\section{Introduction}

Osteoporosis is a common, yet under-diagnosed and undertreated form of musculoskeletal disease [1] which is associated with bone fractures. Approximately one third of men and half of women aged over 60 will sustain a fracture in their lifetime [2]. Experiencing a fragility fracture is associated with significant rates of morbidity and mortality, particularly in the case of hip fractures [3, 4], and places the individual at increased risk of further fractures [5-7]. Osteoporosis is expensive, costing Austra- 
lian taxpayers an estimated A $\$ 7.4$ billion per annum, with over 25,000 healthy lives lost in the financial year 2000-01 [8]. Despite evidence that further fractures may be prevented through patient education and initiation of pharmacological treatment [9-11], self-reported prevalence of osteoporosis is low [12], and many patients who sustain a fracture do not receive any diagnosis, treatment or information regarding osteoporosis [13, 14]; and patients leave hospital with their fracture treated but with osteoporosis uninvestigated, undiagnosed and untreated [13-19]. Therefore, targeting patients who sustain fractures to receive assessment and treatment may reduce refracture rates and subsequently the personal, social and economic burden of osteoporosis.

The study had the following objectives:

- to evaluate the efficacy of an osteoporosis clinical pathway designed to increase rates of follow-up, investigation and treatment for osteoporosis administered in a hospital setting,

- to determine the rates of follow-up by a general practitioner (GP) post-fracture, and subsequent rates of investigation and treatment for osteoporosis,

- to examine the prevalence and source of patient education regarding osteoporosis and osteoporosisrelated services,

- to investigate long-term pharmacological osteoporosis prophylaxis use in patients enrolled in the clinical pathway,

- to determine re-fracture rates in these patients,

- to investigate general practitioners compliance with suggested investigations and treatment plans for these patients post-discharge from acute care.

\section{Materials and methods}

Phase 1: Comparison of patients before and after pathway introduction

\section{Setting and participants}

Participants were patients, aged $\geq 45$ years who had been admitted to the Department of Orthopaedics and Trauma at The Queen Elizabeth Hospital (TQEH), South Australia, between 1 May 2003 and 31 January 2004 for treatment of a fracture sustained with a minimal degree of trauma or subsequent to a fall from standing height or less. The first 48 patients who were admitted after 1 May 2003 received standard care under existing protocols and were treated as the control group, while the 'pathway group' were the first 49 patients enrolled in the osteoporosis clinical pathway after July 2003. Patients were excluded $(\mathrm{N}=7)$ if they had insufficient contact details or were deceased when matched to mortality data. Next of kin were approached if patients had memory loss or dementia. Participants provided written informed consent in accordance with the Declaration of Helsinki (1996). The study was approved by the North Western Adelaide Health Service Ethics of Human Research Committee. Fifty-six patients or next-of-kin $(62 \%$, $56 / 90$ ) consented to participate and completed a questionnaire. The final sample included 28 subjects in each of the control and pathway groups.

\section{Intervention}

Patients meeting inclusion criteria were identified by the clinical pharmacist from computerised hospital lists and case notes. Treatment consisted of oral triple therapy of a bisphosphonate (risedronate $35 \mathrm{mg}$ weekly), calcium supplement (600 mg twice daily) and a Vitamin D supplement (ergocalciferol 1000 units daily). Contraindications to risedronate were gastric ulceration, previous intolerance to a bisphosphonate, or inability to sit upright for 30 minutes after administration. The pharmacist recommended prescription of triple pack therapy by junior medical officers both during admission and on discharge with backup and reinforcement by the orthopaedic physician.

Patients commencing therapy received individual counselling from the clinical pharmacist and were provided with a standard osteoporosis pack. Interpreters were used, and next of kin were contacted in patients with dementia. Each pack contained patient information about osteoporosis and the drugs prescribed, a letter to the general practitioner (GP), an information leaflet for a physiotherapy selfmanagement course, a list of local community falls prevention services, and support products for risedronate. This was presented in a bright orange bag and labelled "Osteoporosis Pack". Patients were offered the opportunity to enrol in the Aventis ACTNOW support programme.

The letter to the patients' GP stated that the patient had been identified as having an osteoporotic fracture and had been provided with an osteoporosis pack. Also included were the date of the X-ray that confirmed the fracture, medications prescribed and suggestions as to the investigation of secondary causes of osteoporosis. It was clearly stated that although the patient had been started on refracture prevention therapy NO investigations of osteoporosis or secondary causes had been undertaken.

\section{Procedure}

Participants completed follow-up questionnaires by home interview or mail 6-12 months following hospital admission for the fracture (mean $44 \pm 13$ weeks). Information 
collected in the questionnaire included the following: demographic information, osteoporosis lifestyle factors, falls and fracture history, family history of fractures or osteoporosis, treatments taken before or initiated following the fracture, osteoporosis-related investigations suggested or undertaken (e.g., bone density test by DXA (dual emission X-ray absorptiometry) serum calcium and vitamin D), follow-up visits to a GP, and whether any information about osteoporosis or osteoporosis-related services had been provided to the patient, as well as the source of the information.

Phase 2: Evaluation of the osteoporosis clinical pathway and General Practitioner feedback

This phase incorporated patients enrolled in the osteoporosis care pathway from February-October 2004. Participants or their proxy (next of kin or nursing home staff member) were interviewed by telephone. Information collected was identical to that collected in phase 1 of the study.

In addition to collecting patient information, a single page questionnaire was sent to all GP's in the catchment area of TQEH by the Western Adelaide Division of General Practice. This requested information about whether individual patients had been seen by the GP post-discharge, and whether investigations and prophylactic medications suggested under the protocol had been arranged.

\section{Statistical analyses}

Chi-square and student t-tests were used to examine sample characteristics. Significance levels of $10 \%$ were used in phase 1 due to the small sample size. Conventional significance levels of 5\% were used in phase 2. Analyses were conducted using SPSS version 12.0.1 (phase 1) and SPSS version 13.0 (phase 2).

\section{Results}

\section{Patient characteristics}

Table 1 shows that in phase 1 of the study patients in the pathway group were significantly older than the control group, less likely to have completed high school, more likely to have had their questionnaire completed by next-ofkin, and, by study design, had shorter time to interview. There were no significant differences between the two groups in regard to gender, place of residence or osteoporosis risk factors. Table 1 also shows that patients in phase 2 of the study were similar to the control group in phase 1 in relation to gender, place of residence and survey respondent.

The most common site of fracture was the hip (See Fig. 1). There were no significant differences in fracture types between pathway and control patients (phase 1) or between patients in phase 1 and phase 2 . In phase 2 of the study, two participants presented with a more than one fracture at time of admission.

\section{Osteoporosis investigations}

Table 2 shows that in phase 1 of the study there were no significant differences between patients enrolled in the pathway and control patients in relation to recommendations for or initiation of osteoporosis investigations.

\section{Osteoporosis medication}

Table 3 shows that in phase 1 of the study significantly more patients enrolled in the care pathway recalled receiving information about medication that could improve bone density or may help in preventing future fractures. Table 3 also shows that this level of recall was maintained in phase 2 of the study. Information about osteoporosis medication was most often provided by the patient's GP;
Table 1 Characteristics of participating patients

${ }^{*} \mathrm{p} \leq 0.1, * * \mathrm{p} \leq 0.05$.

${ }^{a}$ statistically significant difference between phase 1 usual care and phase 1 pathway ${ }^{\mathrm{b}}$ statistically significant difference between phase 1 usual care and phase 2 pathway

\begin{tabular}{llll}
\hline & $\begin{array}{l}\text { Phase 1 Usual Care } \\
\mathrm{n}=28\end{array}$ & $\begin{array}{l}\text { Phase 1 Pathway } \\
\mathrm{n}=28\end{array}$ & $\begin{array}{l}\text { Phase 2 Pathway } \\
\mathrm{n}=65\end{array}$ \\
\hline $\begin{array}{l}\text { Demographics } \\
\text { Age, mean } \pm \mathrm{SD}(\mathrm{yrs})^{\mathrm{a} * *}\end{array}$ & $71.3 \pm 13.7$ & $79.2 \pm 8.4$ & 76.17 \\
Female (\%) $)^{\mathrm{a} * *}$ & 71.4 & 78.6 & 70.8 \\
Did not complete high school (\%) ${ }^{\mathrm{a} *}$ & 57.1 & 78.6 & Not collected \\
Resident of nursing home (\%) & 14.3 & 32.1 & 15.4 \\
Time to interview, mean $\pm \mathrm{SD}(\mathrm{wks})$ & $55.3 \pm 4.4$ & $32.7 \pm 7.1$ & Not collected \\
Next-of-kin as respondent $(\%)^{\mathrm{a} * *}$ & 10.7 & 39.3 & 20.0 \\
Osteoporosis risk factors & & & 4.6 \\
Current smoking $(\%)^{\mathrm{b} * *}$ & 25.0 & 14.3 & 33.8 \\
Previous adult fracture $(\%)$ & 53.6 & 42.9 & 32.3 \\
Family history of fracture $(\%)$ & 35.7 & 17.9 & \\
\hline
\end{tabular}




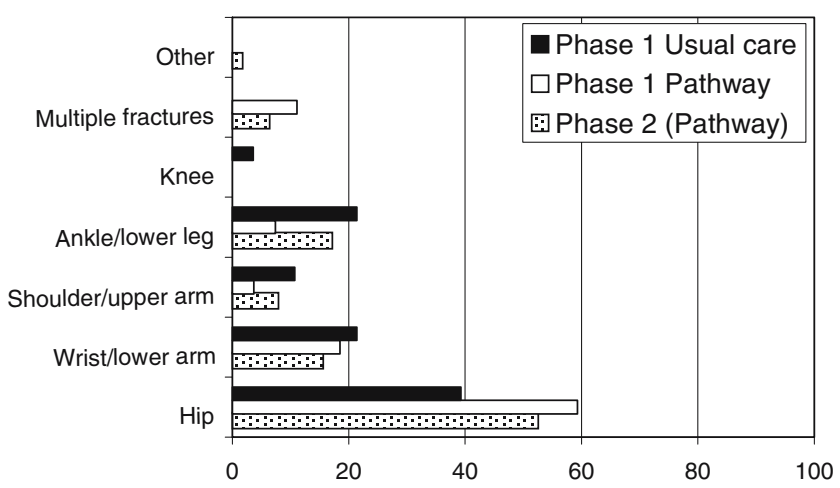

Fig. 1 Site of bone fracture

however, information was also provided by hospital pharmacy staff, an orthopaedic surgeon or from other sources. Patients in the pathway group in phase 1 were more likely than controls to have had osteoporosis medication (bisphosphonate, calcium, vitamin D or hormone replacement therapy) prescribed but this difference was not statistically significant. The majority of patients for whom osteoporosis medication was prescribed, commenced and continued prescribed medications; these numbers were not significantly different between pathway and control groups. The majority of patients in phase 2 were also prescribed osteoporosis medication; most of these patients commenced and continued the medication. Reasons for ceasing medication included the patient not understanding why the medication was needed, the patient reporting experiencing side effects, being unwilling to take the medication or not having a prescription renewed by a GP.

\section{General practitioner follow-up}

The majority of patients consulted a GP following their fracture (See Table 4). Most of these consultations occurred within one month of the patient sustaining the fracture. There was no significant difference between the pathway and usual care groups in to the proportion who visited a GP for follow-up, although patients in phase 2 were more likely to have seen a GP than patients in phase 1. Reasons

Table 2 Osteoporosis investigations

\begin{tabular}{llll}
\hline & $\begin{array}{l}\text { Phase 1 } \\
\text { Usual Care } \\
\mathrm{n}=28\end{array}$ & $\begin{array}{l}\text { Phase 1 } \\
\text { Pathway } \\
\mathrm{n}=28\end{array}$ & $\begin{array}{l}\text { Phase 2 } \\
\text { Pathway } \\
\mathrm{n}=65\end{array}$ \\
\hline Investigation suggested & 32.1 & 39.3 & 29.2 \\
Investigation initiated & 32.1 & 35.7 & 26.1 \\
DXA $^{\text {a }}$ suggested & 25.0 & 28.6 & 27.7 \\
DXA conducted & 25.0 & 21.4 & 29.2 \\
Vitamin D/calcium test suggested & 17.9 & 35.7 & 29.2 \\
Vitamin D/calcium checked & 14.3 & 28.6 & 29.2 \\
\hline
\end{tabular}

${ }^{\mathrm{a}} \mathrm{DXA}=$ dual emission $\mathrm{X}$-ray absorptiometry provided for not seeing a GP post fracture included: patient being discharged to a rehabilitation centre (35\%), patient was a resident of a nursing home $(15 \%)$ and patient belief that they did not need to visit a GP because the fracture had been "fixed" $(25 \%)$

GP responses to emailed questionnaire

No responses to the emailed questionnaire were received from GPs in the hospital catchment area.

\section{Discussion}

The results of this study demonstrate that a clinical pathway administered in a hospital setting is an effective means of increasing rates of treatment and follow-up for osteoporosis in this patient group. The increase in the prescription of osteoporosis medications following pathway introduction seen in phase 1 of the current study is similar to results of an audit of a fracture protocol in Tasmania, Australia [20]. However, we have also demonstrated that this increase in prescription of osteoporosis medications is sustained over time, and that initiating pharmacological treatment in a hospital environment appears to influence the rate that patients fill their scripts and continue to take the medication after discharge. To maintain this level of adherence also requires that the medical practitioners who see the patients after discharge from hospital continue to prescribe these medications. We found that individuals who were enrolled in the osteoporosis clinical pathway were no more likely than controls to receive osteoporosis investigations (e.g., DXA, serum calcium and Vitamin D), but were significantly more likely to have received information about pharmaceutical interventions, and more likely to have actually been prescribed pharmacological osteoporosis prophylaxis. Regardless of whether patients were enrolled in the pathway, adherence to prescribed medications was still high at the follow-up audit.

The results of this study provide support for those of Majumdar et al. [15] who found that treatment delivered via an osteoporosis pathway was associated with an increase in the rates of BMD testing and prescription of osteoporosis treatment. The results also support those of Sidwell et al. [16], who found that there was improvement in the management and treatment of osteoporosis in patients enrolled in a clinical pathway.

The inclusion of patients with dementia through proxy interviewing is a strength of this study. This group is at significant risk of falls and fractures [21], yet are often excluded from research due to the difficulties associated with obtaining consent and collecting information. Face-toface home interviews in phase 1 of the study assisted in a 
Table 3 Osteoporosis treatment, medications and services

\begin{tabular}{|c|c|c|c|c|c|c|}
\hline & \multirow{2}{*}{\multicolumn{2}{|c|}{$\begin{array}{l}\text { Phase } 1 \text { Usual care } \\
\mathrm{n}=28\end{array}$}} & \multirow{2}{*}{\multicolumn{2}{|c|}{$\begin{array}{l}\text { Phase } 1 \text { Pathway } \\
n=28\end{array}$}} & \multirow{2}{*}{\multicolumn{2}{|c|}{$\begin{array}{l}\text { Phase } 2 \text { Pathway } \\
n=65\end{array}$}} \\
\hline & & & & & & \\
\hline & $\mathrm{n}$ & $\%$ & $\mathrm{n}$ & $\%$ & $\mathrm{n}$ & $\%$ \\
\hline $\begin{array}{l}\text { Received information about osteoporosis medication }{ }^{\mathrm{d} * *, \mathrm{e}_{* *}} \\
\text { Source of information about osteoporosis medication }\end{array}$ & 8 & 28.6 & 15 & 53.6 & 33 & 50.8 \\
\hline General practitioner ${ }^{\mathrm{c}}$ & & & 9 & 39.0 & 12 & 36.4 \\
\hline Hospital pharmacy staff & & & 6 & 26.0 & 6 & 18.1 \\
\hline Orthopaedic surgeon & & & 3 & 13.0 & 6 & 18.1 \\
\hline Other sources & & & 3 & 13.0 & 2 & 6.1 \\
\hline Could not remember & & & 2 & 9.0 & 7 & 21.3 \\
\hline Total & & & 23 & 100.0 & 33 & 100.0 \\
\hline Any osteoporosis medication prescribed ${ }^{\mathrm{d} * *, \mathrm{e}_{* * *}, \mathrm{f}_{* * *}}$ & 7 & 25.0 & 15 & 53.6 & 54 & 83.1 \\
\hline Medication commenced $\mathrm{b}^{\mathrm{b}, \mathrm{d} * *}$ & 7 & 100.0 & 13 & 86.7 & 48 & 88.9 \\
\hline Medication continued ${ }^{\mathrm{b}}$ & 6 & 85.7 & 10 & 76.9 & 46 & 95.8 \\
\hline
\end{tabular}

${ }^{\text {a }}$ Pooled data from pathway and control groups in phase 1

${ }^{\mathrm{b}}$ Denominator is $\mathrm{N}$ in column immediately above

${ }^{\mathrm{c}}$ Fisher's exact test used due to small cell sizes

$* * \mathrm{p} \leq 0.05, * * * \mathrm{p} \leq 0.01$.

$\mathrm{d}$ statistically significant difference between phase 1 usual care and phase 1 pathway

${ }^{\mathrm{e}}$ statistically significant difference between phase 1 usual care and phase 2 pathway

${ }^{\mathrm{f}}$ statistically significant difference between phase 1 pathway and phase 2 pathway

reasonable response rate $(62 \%)$ and ensured that patients with hearing difficulties or those too frail to travel were able to participate in the study.

However the study does have several limitations. Due to time and funding restrictions, the sample size in phase 1 proved insufficient to achieve statistically significant differences between groups for some parameters recorded. The retrospective study design relied on participant recall and it is therefore difficult to ascertain the accuracy with which participants may have recalled events that had occurred some time previously. phase 1 participants in both the control and pathway groups anecdotally reported that they were distressed and confused whilst in hospital and had difficulty in recalling any information that they received during their admission. It is also possible that patient's nextof-kin or nursing home staff were unaware of the osteoporosis information provided to fragility fracture patients whilst in hospital. Validity of the study could be improved by comparing participant responses from hospital, GP, or pharmacy records.

The Queen Elizabeth Hospital is located in a low socioeconomic area within Adelaide [22]; therefore these findings may be generalisable to hospital populations with similar socio-economic status and education levels but not necessarily to the wider South Australian population.

Despite efforts to examine the contribution of GPs to the success of the osteoporosis clinical pathway, no responses were received for this section of the study.

In conclusion, despite the number of studies advocating the need for greater efforts in diagnosing and treating osteoporosis in fragility fracture patients, there have not been efficient clinical pathways developed in South Australia to assist in the provision of appropriate investigation and treatment. Evidence suggests that clinical path-
Table 4 General practitioner follow-up

\footnotetext{
${ }^{\text {a }}$ Pooled data from pathway and control groups in phase 1 $* * * \mathrm{p} \leq 0.01$.

${ }^{\mathrm{b}}$ statistically significant difference between phase 1 pathway and phase 2 pathway

${ }^{\mathrm{c}}$ Not multiple response
}

\begin{tabular}{|c|c|c|c|c|c|c|}
\hline & \multirow{2}{*}{\multicolumn{2}{|c|}{$\begin{array}{l}\text { Phase } 1 \text { Usual Care } \\
\mathrm{n}=28\end{array}$}} & \multirow{2}{*}{\multicolumn{2}{|c|}{$\begin{array}{l}\text { Phase } 1 \text { Pathway } \\
\mathrm{n}=28\end{array}$}} & \multirow{2}{*}{\multicolumn{2}{|c|}{$\begin{array}{l}\text { Phase } 2 \text { Pathway } \\
\mathrm{n}=65\end{array}$}} \\
\hline & & & & & & \\
\hline & $\mathrm{n}$ & $\%$ & $\mathrm{n}$ & $\%$ & $\mathrm{n}$ & $\%$ \\
\hline Saw GP following fracture ${ }^{\mathrm{b} * * *}$ & 20 & 71.4 & 16 & 57.1 & 57 & 87.6 \\
\hline \multicolumn{7}{|l|}{ Reasons for not seeing $\mathrm{GP}^{\mathrm{a}, \mathrm{c}}$} \\
\hline Discharge to rehab hospital & & & 7 & 28.6 & & \\
\hline Nursing home resident & & & 3 & 19.0 & & \\
\hline Fracture fixed & & & 5 & 52.4 & & \\
\hline Other & & & 5 & 28.6 & & \\
\hline Total & & & 20 & 100.0 & & \\
\hline
\end{tabular}


ways may be an effective means with which these issues can be addressed; however, there is still a significant problem with failure to adequately follow up patients to ensure that recommendations for investigation and treatment are heeded.

Acknowledgements Dr Catherine Gibb developed the concept of setting up a clinical pathway at TQEH and also provided the impetus and energy for its continuation. Ms Joan Lynch (Osteoporosis South Australia) assisted with early planning of the study. Data collection and analysis for phase 1 was undertaken by Ms Justine Whitham, Project Officer, Osteoporosis South Australia. Data collection and analysis for phase 2 was undertaken by Ms Yvette Marchant, Project Officer, Osteoporosis South Australia. Funding for phase 1 of the study was provided by a "Falls Prevention and Harm Minimisation Innovations" funding grant from the South Australian Department of Health. Funding for phase 2 of the study was provided by Aventis Pharma. Aventis Pharma provided risedronate at a reduced cost to TQEH pharmacy.

Conflict of interest statement Dr. Gibb has received honoraria from Sanofi Aventis and Pfizer.

\section{Appendix}

The Osteoporosis SA Refracture Prevention Pathway Steering Committee consisted of: Ms Gillian Leach, Ms Pauline Kelly (Osteoporosis South Australia), Associate Professor Nick Fazzalari (Division of Tissue Pathology, Institute of Medical and Veterinary Science), Ms Anna Fergusson, Ms Valerie Williams (South Australian Divisions of General Practice), Dr Linda Ferris (Department of Orthopaedics, Modbury Public Hospital), Ms Anne Taylor, Ms Tiffany Gill (Population Research and Outcome Studies Unit, Department of Health), Professor Trevor Hearn, Ms Vanessa Wells (Department of Surgery, Orthopaedic Unit, Repatriation General Hospital), Associate Professor Julian McNeil, Ms Laura Laslett (The University of Adelaide Discipline of Medicine, Modbury Public Hospital), Dr Alice McLennan (Reproductive Medicine Unit (Repromed), The University of Adelaide), Dr Pat Phillips (Endocrinology Unit, The Queen Elizabeth Hospital and Health Service), Professor Malcolm Smith (Department of Medicine, Rheumatology Unit, Repatriation General Hospital).

\section{References}

1. Nguyen TV, Center JR, Eisman JA (2004) Osteoporosis: underrated, underdiagnosed and undertreated. Med J Aust 180: S18-S22

2. Jones G, Nguyen T, Sambrook PN et al (1994) Symptomatic fracture incidence in elderly men and women: the Dubbo
Osteoporosis Epidemiology Study (DOES). Osteoporos Int 4:277-282

3. March LM, Cameron ID, Cumming RG et al (2000) Mortality and morbidity after hip fracture: can evidence based clinical pathways make a difference? J Rheumatol 27:2227-2231

4. Keene GS, Parker MJ, Pryor GA (1993) Mortality and morbidity after hip fractures. BMJ 307:1248-1250

5. Lindsay R, Silverman SL, Cooper C et al (2001) Risk of new vertebral fracture in the year following a fracture. Jama 285:320-323

6. Johansson C, Mellstrom D (1996) An earlier fracture as a risk factor for new fracture and its association with smoking and menopausal age in women. Maturitas 24:97-106

7. Haentjens P, Autier P, Collins J et al (2003) Colles fracture, spine fracture, and subsequent risk of hip fracture in men and women. A meta-analysis. J Bone Joint Surg Am 85-A:1936-1943

8. The Burden of Brittle Bones: Costing Osteoporosis in Australia. Canberra, ACT: Access Economics Pty Ltd.; 2001

9. Cranney A, Guyatt G, Griffith L et al (2002) Meta-analyses of therapies for postmenopausal osteoporosis. IX: Summary of metaanalyses of therapies for postmenopausal osteoporosis. Endocr Rev 23:570-578

10. Hosking D, Adami S, Felsenberg D et al (2003) Comparison of change in bone resorption and bone mineral density with onceweekly alendronate and daily risedronate: a randomised, placebocontrolled study. Curr Med Res Opin 19:383-394

11. McClung MR, Geusens P, Miller PD et al (2001) Effect of risedronate on the risk of hip fracture in elderly women. Hip Intervention Program Study Group. N Engl J Med 344:333-340

12. Phillipov G, Phillips PJ, Leach G et al (1998) Public perceptions and self-reported prevalence of osteoporosis in South Australia. Osteoporos Int 8:552-556

13. Wong PK, Spencer DG, McElduff P et al (2003) Secondary screening for osteoporosis in patients admitted with minimaltrauma fracture to a major teaching hospital. Intern Med J 33:505-510

14. Smith MD, Ross W, Ahern MJ (2001) Missing a therapeutic window of opportunity: an audit of patients attending a tertiary teaching hospital with potentially osteoporotic hip and wrist fractures. J Rheumatol 28:2504-2508

15. Majumdar SR, Rowe BH, Folk D et al (2004) A controlled trial to increase detection and treatment of osteoporosis in older patients with a wrist fracture. Ann Intern Med 141:366-373

16. Sidwell AI, Wilkinson TJ, Hanger HC (2004) Secondary prevention of fractures in older people: evaluation of a protocol for the investigation and treatment of osteoporosis. Intern Med J 34:129-132

17. Myers TA, Briffa NK (2003) Secondary and tertiary prevention in the management of low-trauma fracture. Aust J Physiother 49:25-29

18. Seagger R, Howell J, David H et al (2004) Prevention of secondary osteoporotic fractures-why are we ignoring the evidence? Injury 35:986-988

19. Kamel HK, Hussain MS, Tariq S et al (2000) Failure to diagnose and treat osteoporosis in elderly patients hospitalized with hip fracture. Am J Med 109:326-328

20. Jones G, Warr S, Francis E et al (2005) The effect of a fracture protocol on hospital prescriptions after minimal trauma fractured neck of the femur: a retrospective audit. Osteoporos Int 16:1277-1280

21. Rose S, Maffulli N (1999) Hip fractures. An epidemiological review. Bull Hosp Jt Dis 58:197-201

22. Glover J, Harris K, Tennant S (1999) A social health atlas of South Australia, 2nd edn. Public Health Information Development Unit, the University of Adelaide 